\title{
Therapeutic Effect of Perioperative Mild Hypothermia on Postoperative Neurological Outcomes in Patients with Acute Stanford Type A Aortic Dissection
}

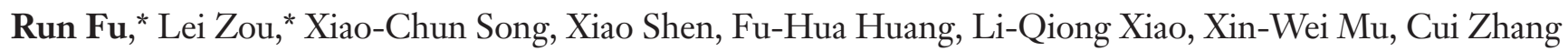 \\ Department of Intensive Care Unit of Nanjing First Hospital, Nanjing Medical University, Nanjing, Jiangsu Province, China
}

\section{ABSTRACT}

Background: Postoperative patients of acute Stanford type A aortic dissection (AAAD) often experience complications consisting of nervous system injury. Mild hypothermia therapy has been proven to provide the therapeutic effect of cerebral protection. We aimed to investigate the therapeutic effects of perioperative mild hypothermia on postoperative neurological outcomes in patients with AAAD.

Methods: A prospective randomized controlled study was conducted on adult patients undergoing aortic dissection surgery between February 2017 and December 2017. Patients in the treatment group underwent mild hypothermia $\left(34^{\circ}\right.$ to $\left.35^{\circ} \mathrm{C}\right)$ immediately after surgery, and in the conventional therapy group, patients were rewarmed to normal body temperature $\left(36^{\circ}\right.$ to $\left.37^{\circ} \mathrm{C}\right)$. Postoperative time to regain consciousness, postoperative serum neuron-specific enolase (NSE) and S-100 $\beta$ levels, cerebral tissue oxygen saturation, presence of delirium or permanent neurological dysfunction, intensive care unit (ICU) and hospital stay duration, and 28-day mortality were compared.

Results: We enrolled 55 patients who underwent AAAD surgery and were randomly allocated into to 2 groups, 27 patients in the treatment group and 28 patients in the conventional therapy group. Compared with the conventional therapy group, postoperative time to regain consciousness was much shorter for patients in the mild hypothermia group (12.65 hours, interquartile range [IQR] 8.28 to 23.82 , versus 25.80 hours, IQR 14.00 to $59.80 ; \mathrm{P}=.02$ ), and the rate of regaining consciousness in 24 hours after surgery was much higher $(74.07 \%$ versus $46.42 \%$; $P=.037)$. At the same time, the ICU stay of patients in the mild hypothermia therapy group was significantly shorter than that in the conventional therapy group $(5.53 \pm 3.13$ versus $9.35 \pm 8.76$ days; $P=.038)$. Cerebral tissue oxygen saturation, incidence of delirium or permanent neurological dysfunction, duration of hospital stay, and 28-day mortality showed no statistical difference. Postoperative serum NSE and S- $100 \beta$ levels increased compared with preoperative baseline values in both groups

Received Fune 11, 2020; received in revised form fuly 29, 2020; accepted fuly 29, 2020.

*These authors contributed equally to this study.

Correspondence: Cui Zhang, Department of Intensive Care Unit of Nanjing First Hospital, Nanjing Medical University, 68 Changle Road, Nanjing, 210006, Fiangsu Province, China; 86-18951670283 (e-mail: zhangcui06123@163.com).
$(P<.05)$, and the serum NSE levels of patients in the mild hypothermia therapy was significantly lower than the conventional therapy group 1 hour $(P=.049)$ and 6 hours $(P=.04)$ after surgery. There was no difference in the chest drainage volume or shivering between the 2 groups 24 hours after surgery.

Conclusions: Perioperative mild hypothermia therapy is able to significantly reduce brain cell injury and shorten the postoperative time to regain consciousness, thus improving the neurological prognosis of patients with AAAD.

\section{INTRODUCTION}

Aortic dissection, a macrovascular disease, is characterized by poor prognosis and high mortality. Once diagnosed, according to the DeBakey or Stanford classification systems, different management strategies are made, including surgical therapy and interventional therapy [Nienaber 2015]. Acute Stanford type A aortic dissection (AAAD) requires surgical therapy. During aortic arch surgery, circulatory arrest (CA) and unilateral cerebral perfusion are needed, which lead to transient ischemia and subsequent ischemia reperfusion injury of organs and systems, including acute lung injury, acute myocardial damage, acute kidney injury, and especially

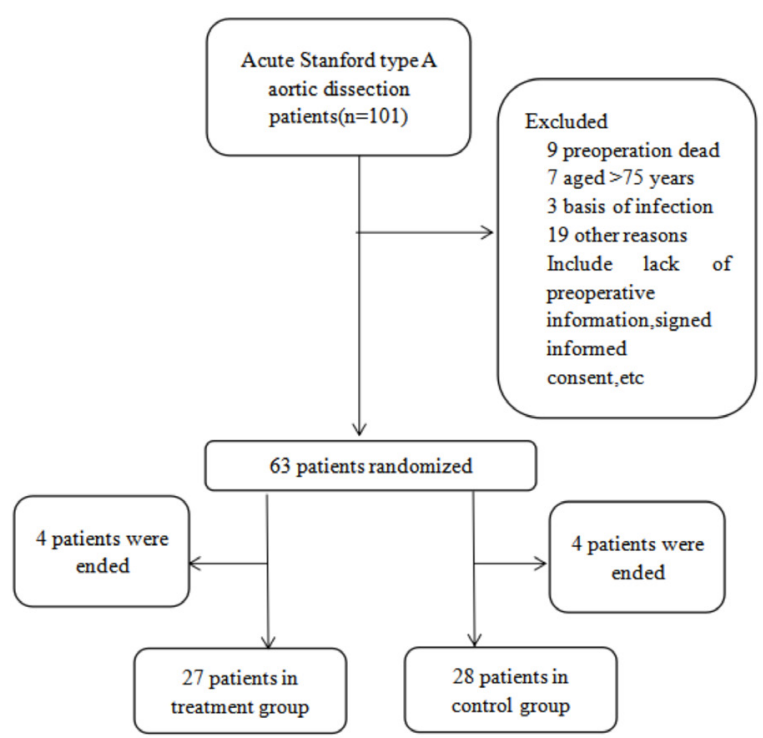

Figure 1. Flow diagram of participants. 
Table 1. Baseline Characteristics of the Patients*

\begin{tabular}{lccc}
\hline Characteristic & $\begin{array}{c}\text { Treatment Group } \\
(\mathrm{n}=27)\end{array}$ & $\begin{array}{c}\text { Control Group } \\
(\mathrm{n}=28)\end{array}$ & $P$ \\
\hline Sex (M/F) & $23 / 4$ & $20 / 8$ & .22 \\
Age (y) & & & .49 \\
$\quad$ Mean $\pm \mathrm{SD}$ & $53 \pm 12$ & $51 \pm 9$ & \\
$\quad$ Range & 27 to 75 & 34 to 72 & \\
Body weight $(\mathrm{kg})$ & $74 \pm 12$ & $75 \pm 14$ & .84 \\
BMl $\left(\mathrm{kg} / \mathrm{m}^{2}\right)$ & $25 \pm 2.7$ & $26 \pm 4$ & .47 \\
Drinking history & $8(29.6)$ & $7(25.0)$ & .70 \\
Hypertension & $25(92.5)$ & $24(85.7)$ & .42 \\
Coronary heart disease & 0 & $2(7.1)$ & .61 \\
Diabetes mellitus & $2(7.4)$ & $2(7.1)$ & .97 \\
APACHE II score & $16 \pm 5$ & $15 \pm 3$ & .54 \\
\hline
\end{tabular}

*Data are mean \pm standard deviation (SD) or $\mathrm{n}(\%)$ unless noted otherwise.

nervous system injury, the incidence of which is reported to reach up to $47.5 \%$ [Amir 2005]. Nervous system injury affects the prognosis of AAAD patients.

The probable mechanisms of nervous system injury in postoperative AAAD patients include several factors. First, systematic inflammation may be important. Acute aortic dissection (AAD) itself is one kind of inflammatory disease, and during the operation, cardiopulmonary bypass (CPB) and ischemia reperfusion injury also result in the release of a series of cytokines and inflammatory mediators. Second, in the perioperative period, many kinds of severe stress, such as pain and surgical trauma, activate the sympathetic-adrenal medulla axis; stress hormones are also released, including oxygen radicals, which damage the brain. During the operation, deep hypothermic $\mathrm{CPB}$ is used to protect organ function. When rewarming after $\mathrm{CPB}$, if the speed of rewarming is too fast or the temperature too high, neurons are damaged. Other factors may be involved as well.

Mild hypothermia therapy $\left(30^{\circ}\right.$ to $\left.35^{\circ} \mathrm{C}\right)$ is widely used for cerebral protection after cardiac arrest, cerebral infarction, cerebral hemorrhage, cerebral trauma, and so on. The possible mechanisms include reducing cerebral metabolism [Berko 2016], attenuating the inflammatory response [Zhi 2016], reducing the release of oxygen radicals, alleviating blood-brain barrier disruptions, and reducing brain edema [Blomquist 2011].

At present, AAAD surgery involves deep hypothermic circulatory arrest (DHCA), which can reduce cerebral metabolic rate and prolong cerebral ischemia time, as an important strategy of brain protection. At the same time, brain ischemiareperfusion injury still occurs after surgery. Previous research has shown that fast rewarming is associated with worse cognitive performance [Ali 2000]. Therefore, we investigated the effects of perioperative mild hypothermia intervention on neurological function and prognosis in patients with AAAD.

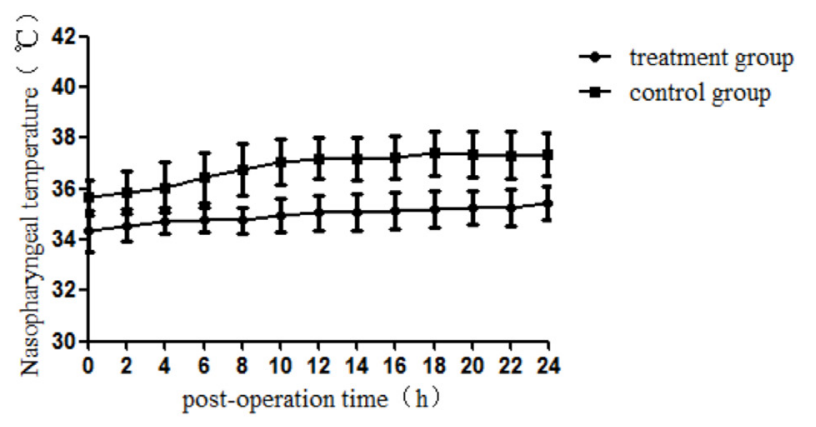

Figure 2. Changes of nasopharyngeal temperature after operation in 2 groups of patients. In the treatment group, nasopharyngeal temperature was maintained at $34^{\circ}$ to $35^{\circ} \mathrm{C}$, and the patients in the control group were rewarmed to $36^{\circ} \mathrm{C}$.

\section{METHODS}

\section{Patient Selection}

This prospective study, which was approved by the ethics committee of Nanjing First Hospital, was performed in a single-center intensive care unit (ICU) in Nanjing First Hospital, Jiangsu Province, China. Between February 2017 and December 2017, 55 patients undergoing AAAD surgery were enrolled. Patients (or relatives) enrolled in this study signed informed consent. Inclusion criteria were age $>18$ and $<75$ years, AAAD involving the aortic arch, confirmed by computed tomography angiography (CTA) and echocardiography, and requiring surgical treatment. The operation was performed under DHCA. Exclusion criteria were immediately death after surgery, history of nervous system disease or mental illness, long-term use of hormones or immunosuppressive agents, confirmed infection, and history of malignant tumors, other immune diseases, or organ transplants. Mild hypothermia therapy was stopped in the case of adverse events.

\section{Study Design}

After AAAD diagnosis, patients were divided into the mild hypothermia therapy group (treatment group) and the conventional therapy group (control group) by numerical randomization. Before the operation, the patients were admitted to the ICU and received the same treatment and preoperative preparation, such as sedation, analgesia, and controlling blood pressure and heart rate. Preoperative blood samples were collected.

The same group of experienced anesthetists and surgeons performed the operations. All patients underwent Sun's procedure. All operations used general anesthesia and extracorporeal circulation via axillary vein and right atrium. Temperature was measured using a nasopharyngeal probe. When nasopharyngeal temperature dropped to $26^{\circ} \mathrm{C}$, aortic arch surgery was begun, and unilateral cerebral perfusion was administered by right subclavian artery. During the operation, $20 \mathrm{mg} / \mathrm{kg}$ methylprednisolone was given to all patients. After DHCA, patients in the treatment group were gradually 
Table 2. Intraoperative Data*

\begin{tabular}{|c|c|c|c|}
\hline Surgical procedure duration (min) & $541 \pm 106$ & $530 \pm 73$ & .66 \\
\hline Aortic cross-clamping time (min) & $115 \pm 26$ & $117 \pm 17$ & .73 \\
\hline DHCA time (min) & $16.92 \pm 3.40$ & $19.08 \pm 4.90$ & .26 \\
\hline Blood loss (mL) & $1200(1000$ to 1400$)$ & 1900 (1300 to 2300$)$ & .11 \\
\hline Blood transfusions $(\mathrm{mL})$ & $4691 \pm 1180$ & $4865 \pm 2552$ & .73 \\
\hline Urine volume (mL) & $2090 \pm 1627$ & $2063 \pm 1027$ & .93 \\
\hline
\end{tabular}

*Data are mean \pm standard deviation or mean (range).

Table 3. Neurological Function and Prognostic Indicators

\begin{tabular}{|c|c|c|c|}
\hline Regained consciousness $24 \mathrm{~h}$ after surgery & $20(74.07)$ & $13(46.42)$ & $.037 \dagger$ \\
\hline Delirium & $10(37.03)$ & $13(46.42)$ & .48 \\
\hline Cerebrovascular accident & $2(7.40)$ & $3(10.71)$ & .67 \\
\hline Total hospital stay $(d)$ & $20.40 \pm 7.45$ & $22.78 \pm 9.60$ & .31 \\
\hline 28-day mortality & 2 & 2 & .97 \\
\hline Chest drainage volume $24 \mathrm{~h}$ after surgery $(\mathrm{mL})$ & $617 \pm 400$ & $741 \pm 571$ & .359 \\
\hline Shivering $24 \mathrm{~h}$ after surgery & $1(3.70)$ & 0 & .327 \\
\hline
\end{tabular}

*Data are $\mathrm{n}(\%)$, mean (range), or mean \pm standard deviation.

†Statistically significant.

rewarmed to a nasopharyngeal temperature of $34^{\circ} \mathrm{C}$, and patients in the control group were gradually rewarmed to a nasopharyngeal temperature of $36^{\circ} \mathrm{C}$.

After surgery, patients were admitted to the ICU and received standard management, including gastric mucosa protection, maintenance of hemodynamic stability and homeostasis by fluid therapy and vasoactive agents, oxygen administration, and antibiotic prophylaxis. Treatment group patients were maintained at a nasopharyngeal temperature of $34^{\circ}$ to $35^{\circ} \mathrm{C}$ by ice blankets, cooling machine, and ice cap; control group patients stayed at $36^{\circ} \mathrm{C}$. Target temperature was maintained for 24 hours after surgery in both groups. There was no difference in the remaining treatment between the 2 groups. After the patients regained consciousness, myodynamia was recovered, hemodynamics became stable, and arterial blood gas results were approved, tracheal intubations were removed. When vital signs were stable for 24 hours after extubation, patients were transferred to the general ward.

\section{Data Collection}

Clinical baseline values were collected retrospectively at ICU admission, including sex, age, body weight, body mass index (BMI), Acute Physiology and Chronic Health Evaluation II (APACHE II) score, basic disease, and so on. During the operation, operating time, cardiopulmonary bypass time, aortic cross-clamping time, DHCA time, selective antegrade cerebral perfusion (SACP) time, blood loss, and blood transfusions were recorded. After surgery, neurological function and prognostic indicators were evaluated and recorded, including cerebral oxygen saturation, recovery time, incidence of delirium and cerebrovascular accidents, mechanical ventilation time, duration of ICU and hospital stays, and 28-day mortality.

Blood samples collected at ICU admission and 1, 6, 12, and 24 hours after the operation were clotted at room temperature for 30 minutes. Samples were then centrifuged for 10 minutes at $3000 \mathrm{~g}$, and serum was collected in $2.0-\mathrm{mL}$ Eppendorf tubes and stored at $-80^{\circ} \mathrm{C}$ until batchwise analysis. We measured 


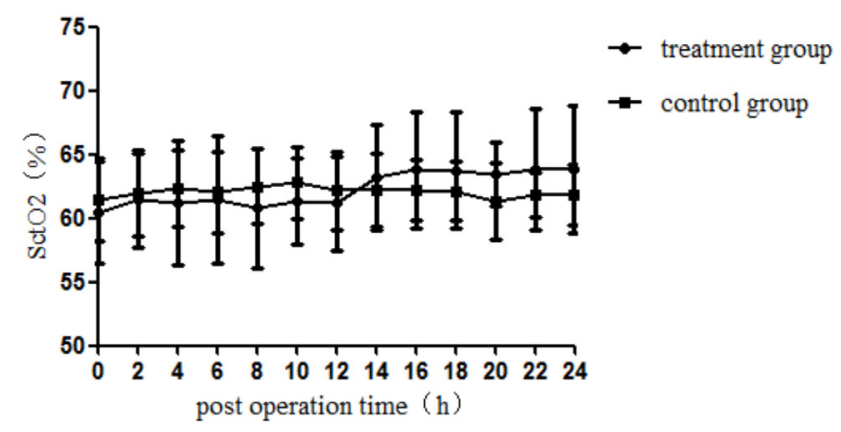

Figure 3. Changes of cerebral oxygen saturation after operation in 2 groups of patients. Cerebral oxygen saturation rises in the treatment group when viewing the situation as a whole; the conventional therapy group showed no fluctuation, but there was no statistically significant difference.

both serum neuron-specific enolase (NSE) and S100ß levels by enzyme-linked immunosorbent assay (ELISA, R\&D Systems, Minneapolis, MN, or SenBeiJia Biotechnology, NanJing, China) according to the manufacturer's protocol. Increased chest drainage volume during treatment or shivering 24 hours postoperatively were recorded as adverse events.

\section{Statistical Analysis}

Statistical analysis used SPSS 22.0 software (IBM Analytics, Armonk, NY). The presentation of data includes value, percentage, median, and interquartile range (IQR). Statistical assessment of classified variables adopts the chisquare test, and the statistical assessment of continuous variables adopts the t test. All charts were produced in Prism 7.0 software (GraphPad, San Diego, CA). $P<.05$ (bilateral) was considered statistically significant.

\section{RESULTS}

From February 2017 to December 2017, 101 patients admitted to the ICU of Nanjing First Hospital were enrolled in this study. Of these patients, 63 were eligible, including 8 patients who subsequently withdrew after randomization. This resulted in an intent-to-treat population of 55 patients ( 27 in the treatment group and 28 in the control group). The screening and selection process are shown in Figure 1. Changes in nasopharyngeal temperature of patients in both groups are shown in Figure 2.

Demographic data and clinical characteristics of the 2 groups were similar (Table 1). Hypertension was the most common underlying disease in these patients $(25$ in the treatment group and 24 in the control group; $P=.42)$. The illness severity of these patients was very high (APACHE II score 16 \pm 5 in the treatment group and $15 \pm 3$ in the control group; $P=.54)$. During the operation, there were no differences in surgical procedure duration, cardiopulmonary bypass time, aortic cross-clamping time, DHCA time, SACP time, or blood loss, blood transfusion, or urine volumes (Table 2).
Table 4. Preoperative and Postoperative Serum NSE Levels*

\begin{tabular}{lccc}
\hline NSE $(\mathrm{pg} / \mathrm{mL})$ & $\begin{array}{c}\text { Treatment Group } \\
(\mathrm{n}=27)\end{array}$ & $\begin{array}{c}\text { Control Group } \\
(\mathrm{n}=28)\end{array}$ & $P$ \\
\hline Baseline & $3313 \pm 2143$ & $4877 \pm 4020$ & .070 \\
Postoperative & & & \\
$1 \mathrm{~h}$ & $5759 \pm 2672$ & $7762 \pm 4406$ & $.049 \dagger$ \\
$6 \mathrm{~h}$ & $5956 \pm 2673$ & $6813 \pm 3243$ & .291 \\
$12 \mathrm{~h}$ & $5689 \pm 3424$ & $5644 \pm 3315$ & .961 \\
$24 \mathrm{~h}$ & $4805 \pm 2149$ & $5211 \pm 3860$ & .673 \\
\hline
\end{tabular}

*Data are mean \pm standard deviation.

†Statistically significant.

The curves of cerebral oxygen saturation in the treatment and control groups 24 hours after surgery are shown in Figure 3 . In the control group, the cerebral oxygen saturation curve remained slightly fluctuating around $62 \%$, with no significant change. Patients in the treatment group showed an increase in cerebral oxygen saturation after hour 12 compared with the control group.

During the first 24 hours after surgery, more patients in the mild hypothermia therapy group regained consciousness (20 of $27,74.07 \%$, in the treatment group versus 13 of 28 , $46.42 \%$, in the control group; $P=.037$ ). Time to regain consciousness was 12.7 hours (IQR 8.10 to 23.80 ) in the treatment group versus 25.8 hours (IQR 14.00 to 59.80) in the control group $(P=.02)$. Duration of ICU stay also was significantly different between the 2 groups (treatment group, 5.53 \pm 3.13 , versus control group, $9.35 \pm 8.76 ; P=.038$ ). However, no significant differences were observed between the 2 groups for other parameters, including incidence of delirium, rate of cerebrovascular accident, duration of hospital stay, or 28-day mortality (Table 3).

Preoperative NSE was $3313 \pm 2143 \mathrm{pg} / \mathrm{mL}$ in the treatment group and $4877 \pm 4020 \mathrm{pg} / \mathrm{mL}$ in the control group $(P=.07)$. At 1 hour after surgery, NSE reached a level of $5759 \pm 2672 \mathrm{pg} / \mathrm{mL}$ in the treatment group, significantly lower than the control group $(7762 \pm 4406 \mathrm{pg} / \mathrm{mL} ; P=.049)$ (Table 4). There was no statistical significance at the other 3 time points. Compared with NSE levels at baseline, postoperative NSE was significantly increased in both groups at all time points. Preoperative S100 $\beta$ was $27 \mathrm{ng} / \mathrm{L}$ (IQR 8 to 40) in the treatment group and $18 \mathrm{ng} / \mathrm{L}$ (IQR 11 to 37 ) in the control group $(P=.65)$. At 6 hours after surgery, both groups reached peak values. However, S100 $\beta$ in the treatment group was significantly less than in the control group. There were no significant differences at the other time points.

\section{DISCUSSION}

AAAD requires surgery with DHCA. DHCA is an important brain-protective strategy, because it can reduce cerebral 
Table 5. Preoperative and Postoperative Serum S100 $\beta$ Levels*

\begin{tabular}{llll}
\hline S100 $\beta(\mathrm{ng} / \mathrm{L})$ & $\begin{array}{c}\text { Treatment Group } \\
(\mathrm{n}=27)\end{array}$ & $\begin{array}{c}\text { Control Group } \\
(\mathrm{n}=28)\end{array}$ & $P$ \\
\hline $\begin{array}{l}\text { Baseline } \\
\text { Postoperative } \\
1 \mathrm{~h}\end{array}$ & $27(8$ to 40$)$ & $18(11$ to 37$)$ & 0.65 \\
$6 \mathrm{~h}$ & $72(51$ to 147$)$ & $106(58$ to 182$)$ & .14 \\
$12 \mathrm{~h}$ & $91(49$ to 136$)$ & $114(42$ to 206$)$ & $.04^{*}$ \\
$24 \mathrm{~h}$ & $83(50$ to 140$)$ & $81(33$ to 149$)$ & .12 \\
\hline
\end{tabular}

*Data are mean (range).

†Statistically significant.

metabolic rate and prolong cerebral ischemia time. The data clearly indicate that hyperthermia should be avoided in the perioperative period, necessitating careful rewarming strategies if hypothermia is used during surgery [Grigore 2009]. Related studies have shown that rapid rewarming can exacerbate ischemic neuronal damage and accelerate the death of neurons [Grigore 2002; Hori 2015]. In contrast, slow rewarming can reduce nerve cell damage, which is conducive to brain protection. Prolonged use of cooling can also prevent body temperature rebound during subhypothermia treatment. Our study results show that patients in the mild hypothermia group had a significantly shorter recovery time after surgery and a reduced ICU stay.

Cerebral hypoxia, as a result of injury, is recognized as one of the most important causes of secondary insults in patients undergoing aortic dissection surgery. Many studies on local brain tissue oxygenation have been published, and the importance of cerebral oxygenation monitoring has been increasingly recognized in neonatal intensive care. Given that it can reflect brain oxygenation [Dix 2017], we monitored cerebral oxygen saturation $\left(\mathrm{rSO}_{2}\right.$ ) for 24 hours after surgery in both groups. Our data suggest that cerebral oxygen saturation rises after mild hypothermia therapy when viewing the situation as a whole; however, the conventional therapy group showed no fluctuation (Figure 3). Our study indicates that mild hypothermia therapy can reduce cerebral metabolism to some extent, but more research is needed.

Neuron-specific enolase is a peripheral blood marker of neuronal injury. It is a $78-\mathrm{kD}$ dimeric $\gamma$-isoenzyme of the glycolytic enzyme enolase, mainly localized in the cytoplasm of neurons [Yokobori 2013]. Normally, NSE cannot be secreted into extracellular fluids. When neurons are damaged, NSE can be expressed at high levels in the brain and released into the blood, indicating the degree of neuron damage [Reiber 2003]. Its half-life is approximately 24 hours. NSE has been proven as a marker to evaluate brain damage and outcome in cerebral infarction [Schaarschmidt 1994], subarachnoid hemorrhage [Persson 1987], and diffuse axonal injury [Chabok 2012]; furthermore, it helps predict early prognosis after traumatic brain injury [Meric
2010] and cardiac arrest [Stammet 2015]. Our data indicate that, after surgery, NSE levels significantly increased versus before the operation in both groups, as in previous studies [Bonacchi 2006]. At 1 hour after surgery, NSE levels were significantly lower in the mild hypothermia therapy group. This suggests that brain damage from the surgical procedure of AAAD could be alleviated by early mild hypothermia therapy.

Among brain damage biomarkers, $\mathrm{S} 100$ protein is a lowmolecular-weight (9- to $13-\mathrm{kDa}$ ), $\mathrm{Ca}^{2+}$-binding protein that primarily participates in neuronal differentiation, proliferation, and apoptosis [Donato 1999]. In the central nervous system, the S100 $\beta$ subtype is widely studied [Schafer 1996]. S100 $\beta$ is secreted by oligodendrocytes and astrocytes [Milleit 2016]. As a small protein, it can pass through the blood-brain barrier. When nerve cells are damaged because of various pathological conditions, or when blood-brain barrier permeability increases significantly, serum levels of $S 100 \beta$ can increase abnormally [Zhi 2016]. Thus, S100 $\beta$ can be regarded as a useful and sensitive biomarker for neuron damage that predicts the extent of brain injury [Blomquist 2011; Ali 2000]. In our study, we found that the $S 100 \beta$ level rose significantly in both groups after the operation. At 6 hours after surgery, levels in the treatment group were significantly lower than in the control group. This result also suggests that mild hypothermia therapy has a role to play in preventing brain injury.

Coagulation dysfunction is one of the most common and severe complications of mild hypothermia [Emmert 2018]. Stein et al [2017] reviewed 98 patients undergoing aortic surgery to evaluate the effect of intraoperative deep hypothermia on postoperative bleeding and found that there was no significant correlation between intraoperative hypothermia and postoperative bleeding, suggesting that intraoperative hypothermia did not increase the risk of bleeding. Similarly, in our study, no significant difference was found in blood loss and 24-hour postoperative chest drainage volume between the treatment group and the control group. These results suggest that perioperative hypothermia intervention did not increase the risk of intraoperative and postoperative bleeding in AAAD patients.

There are some limitations of our study. First, this was a single-center study, and the sample size was relatively small, mainly because of the low incidence of acute dissection. Few studies have focused on mild hypothermia therapy in acute dissection patients, so the present study can still be a useful reference for mild hypothermia therapy in this population and provide a basis for further studies. Second, our study did not monitor and assess long-term neurological outcomes, but we will continue to follow up with these patients.

\section{CONCLUSION}

Perioperative mild hypothermia therapy is able to significantly reduce brain cell injury and shorten the time to regain consciousness and thus improve the neurological prognosis of patients with AAAD. 


\section{REFERENCES}

Ali MS, Harmer M, Vaughan R. 2000. Serum S100B protein as a marker of cerebral damage during cardiac surgery. Br JAnaesth 85:287-298.

Amir G, Ramamoorthy C, Riemer RK, et al. 2005. Neonatal brain protection and deep hypothermic circulatory arrest: Pathophysiology of ischemic neuronal injury and protective strategies. Ann Thorac Surg 80:1955-1964.

Berko M, Stefan S, Matthias R, et al. 2016. Serum S100B protein is specifically related to white matter changes in schizophrenia. Front Cell Neurosci 10:33.

Blomquist S, Johnsson P, Luhrs-C, et al. 2011. The appearance of S-100 protein in aerum during and immediately after cardiopulmonary bypass surgery: A possible marker for cerebral injury. Cardiothorac Vasc Anesth 11:699-703.

Bonacchi M, Prifti E, Maiani M, et al. 2006. Does off-pump coronary revascularization reduce the release of the cerebral markers, S-100 $\beta$ and NSE? Heart Lung Circ 15:314-319.

Chabok SY, Moghadam AD, Saneei Z, et al. 2012. Neuron-specific enolase and $\mathrm{S} 100 \mathrm{BB}$ as outcome predictors in severe diffuse axonal injury. $\mathrm{J}$ Trauma Acute Care Surg 72:1654-1657.

Dix LM, Van Bel F, Lemmers PM. 2017. Monitoring cerebral oxygenation in neonates: An update. Front Pediatr 5:46.

Donato R. 1999. Functional roles of S100 proteins, calcium-binding proteins of the EF-hand type. Biochim Biophys Acta 1450:191-231.

Emmert A, Gries G, Wand S, et al. 2018. Association between perioperative hypothermia and patient outcomes after thoracic surgery: A single center retrospective analysis. Medicine 97:e0528.

Grigore AM, Grocott HP, Mathew JP, et al. 2002. The rewarming rate and increased peak temperature alter neurocognitive outcome after cardiac surgery. Anesth Analges 94:4-10.

Grigore AM, Murray CF, Ramakrishna H, et al. 2009. A core review of temperature regimens and neuroprotection during cardiopulmonary bypass: Does rewarming rate matter ? Anesth Analges 109:1741-1751.
Hori D, Everett AD, Lee JK, et al. 2015. Rewarming rate during cardiopulmonary bypass is associated with release of glial fibrillary acidic protein. Ann Thorac Surg 100:1353-1358.

Meric E, Gunduz A, Turedi S, et al. 2010. The prognostic value of neuron-specific enolase in head trauma patients. J Emerg Med 38:297-301.

Milleit B, Smesny S, Rothermundt M, et al. 2016. Serum S100B protein is specifically related to white matter changes in schizophrenia. Front Cell Neurosci 10:33.

Nienaber CA, Clough RE. 2015. Management of acute aortic dissection. Lancet 385:800-811.

Persson L, Haardemark H-G, Gustafsson J. 1987. S-100 protein and neuron-specific enolase in cerebrospinal fluid and serum: Markers of cell damage in human central nervous system. Stroke 18:1-8.

Reiber H. 2003. Proteins in cerebrospinal fluid and blood: Barriers, CSF flow rate and source-related dynamics. Restor Neurol Neurosci 21:1-19.

Schaarschmidt H, Prange H, Reiber H. 1994. Neuron-specific enolase concentrations. Stroke 25:558-565.

Schafer BW, Heizmann CW. 1996. The S100 family of EF-hand calcium-binding proteins: Functions and pathology. Trends Biochem Sci $21: 134-140$

Stammet P, Collignon O, Hassager C, et al. 2015. Neuron-specific enolase as a predictor of death or poor neurological outcome after out-ofhospital cardiac arrest and targeted temperature management at $33^{\circ} \mathrm{C}$ and $36^{\circ} \mathrm{C}$. J Am Coll Cardiol 65:2104-2114.

Stein LH, Rubinfeld G, Balsam LB, et al. 2017. Too cold to clot? Does intraoperative hypothermia contribute to bleeding after aortic surgery? Aorta 5:106-116.

Yokobori S, Hosein K, Burks S, et al. 2013. Biomarkers for the clinical differential diagnosis in traumatic brain injury: A systematic review. CNS Neurosci Therapeut 19:556-565.

Zhi XL, Li CY, Xue M, et al. 2016. Changes in cognitive function due to combined propofol and remifentanil treatment are associated with phosphorylation of tau in the hippocampus, abnormal total water and calcium contents of the brain, and elevated serum S100ß levels. Eur Rev Med Pharmacol Sci 20:2156-2162. 